\title{
Active pulmonary tuberculosis manifesting with idiopathic thrombocytopenic purpura: a rare presentation
}

\begin{abstract}
A 17-year-old girl presented with a 3-day history of epistaxis, vaginal bleeding and petechiae over the lower extremities. The patient had been feeling unwell with productive cough, fever, chills, poor appetite and weight loss for 2 months. Laboratory findings revealed anemia and thrombocytopenia, whereas bone marrow examination was unremarkable. She was diagnosed as having idiopathic thrombocytopenic purpura (ITP) in association with active tuberculosis (TB). The patient was treated with intravenous immunoglobulin (IVIg) and corticosteroid along with anti-TB drugs. During the follow-up period there was no recurrence of thrombocytopenia or TB. It is important to consider TB in the differential diagnosis of ITP, particularly in high TB-burden areas.
\end{abstract}

Keywords: idiopathic thrombocytopenic purpura; tuberculosis.

[Braz J Infect Dis 2010;14(6):639-640]@Elsevier Editora Ltda.

\section{TO THE EDITOR}

A 17-year-old girl presented with a 3-day history of epistaxis, vaginal bleeding and petechiae over the lower extremities. The patient had been feeling unwell with productive cough but no hemoptysis, fever, chills, poor appetite and weight loss for 2 months. She had no known blood disorder. All vital signs were normal apart from hypotension (105/65 mmHg). The patient had a pale appearance with multiple petechiae over the lower extremities. There were diminished breath sounds with scattered crackles over the right lower chest. Her physical examina- tion was otherwise unremarkable. Laboratory findings revealed low hemoglobin $(\mathrm{Hb})$ of $7.8 \mathrm{~g} / \mathrm{dL}$, low platelet count of $36 \times 10^{3} / \mu \mathrm{L}$ and high ESR of $115 \mathrm{~mm} / \mathrm{hr}$. A bone marrow examination was unremarkable. The sputum was positive for AFB on three consecutive smear examinations. A PCR analysis for $M y$ cobacterium tuberculosis (MTb) turned out positive. The isolated MTb was susceptible to both isoniazid and rifampicin. The rest of her laboratory tests were normal. A chest $\mathrm{X}$-ray (CXR) and a computed tomography (CT) revealed mediastinal and right hilar lymphadenopathy with consolidation of the right middle and lower lobes (Figures 1

Figure 1: Chest X-ray showing mediastinal and right hilar lymphadenopathy with consolidation of the right middle and lower lobes.

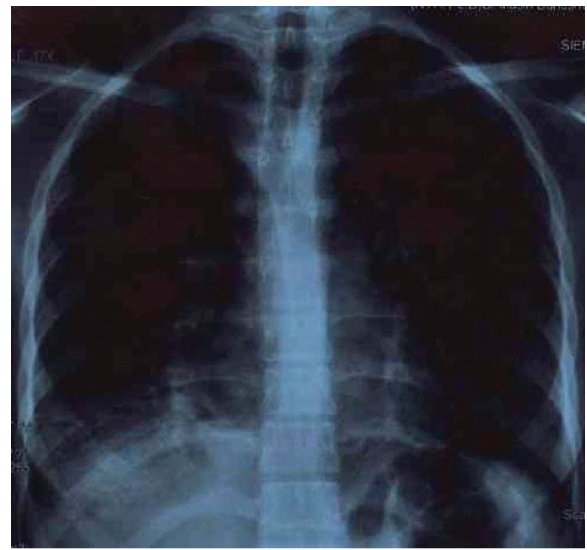

Authors

Payam Tabarsi ${ }^{1}$

Muayad Aghali Merza

Majid Marjani ${ }^{1}$

${ }^{1}$ Infectious Diseases Doctor, Mycobacteriology Research Center (MRC),

National Research Institute of Tuberculosis and Lung

Diseases (NRITLD),

Shaheed Beheshti

University of Medical

Sciences, Darabad, Tehran, Iran.

Submitted on: 07/24/2010 Approved on: 07/29/2010

Correspondence to: Muayad Aghali Merza Shahid Beheshti University of Medical Sciences,

Shaheed Bahonar Ave, Darabad,

P.O. Box 19575/154, Tehran 19556, Iran. Phone: +98 218408275 Fax: +98 212285777 E-mail: muayadfaily@ yahoo.com

We declare no conflict of interest. 
and 2). CT also showed tree-in-bud pattern of the right upper lobe. Based on the clinical, laboratory and radiological findings, she was diagnosed as having idiopathic thrombocytopenic purpura (ITP) in association with active tuberculosis (TB). Based on the American Society of Hematology, ITP is a diagnosis of exclusion, which is made by confirming the presence of isolated thrombocytopenia and by excluding concurrent causes of thrombocytopenia. ${ }^{1}$ The association between TB and ITP is exceedingly uncommon..$^{2-4}$ Although the mechanism of this phenomenon is unclear, MTb could nonspecifically activate a clone of B lymphocytes, which might produce anti-platelet autoantibodies. ${ }^{3}$ The patient was treated with high-dose intravenous immunoglobulin (IVIg) (20 g/day, 5 days) and corticosteroid along with anti-TB drugs. Despite discontinuing IVIg and corticosteroid during the treatment, platelet count remained normal; this strongly suggests the causative role of $\mathrm{MTb}$ in inducing ITP. Follow-up period showed recurrence of neither thrombocytopenia nor TB. In conclusion, it is important to consider TB in the differential diagnosis of ITP, particularly in high TB-burden areas.

Figure 2: Thoracic computed tomography (CT). (A) Demonstrating tree-in-bud pattern of the right upper lobe and parenchymal consolidation.(B) Showing mediastinal and right hilar lymphadenopathy.
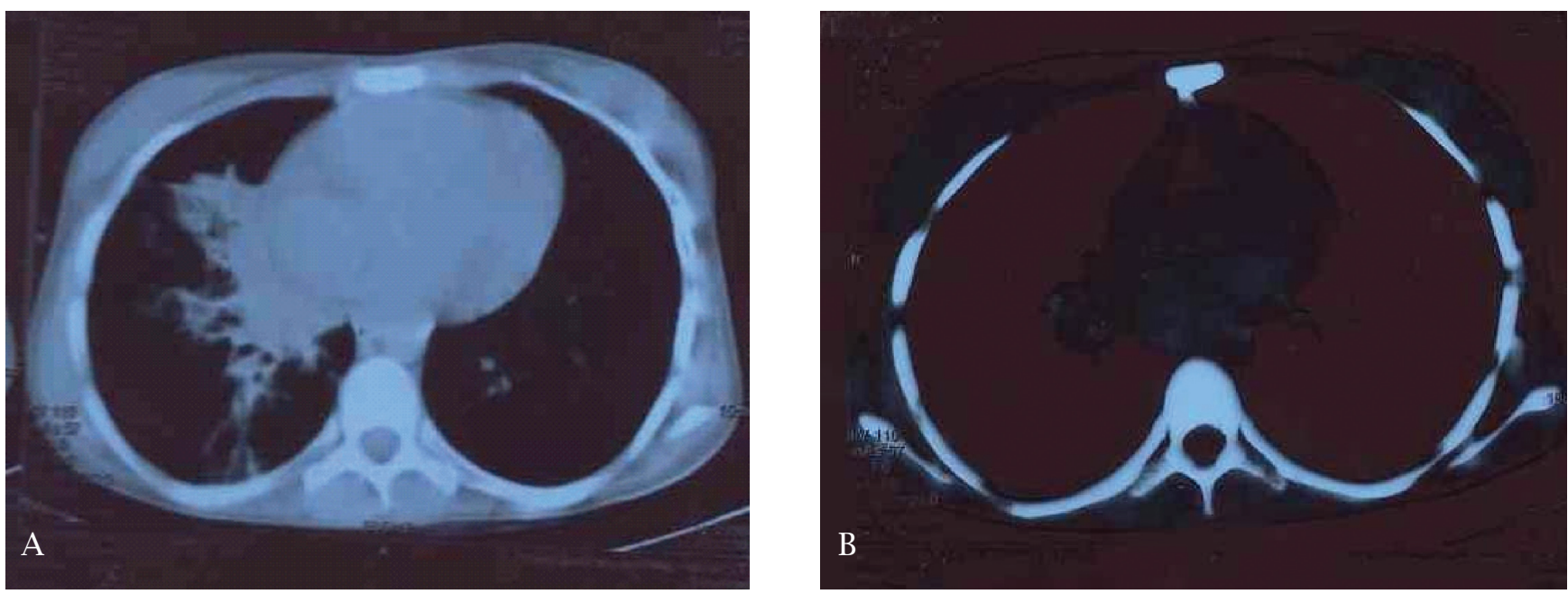

\section{REFERENCES}

1. Diagnosis and treatment of idiopathic thrombocytopenic purpura: recommendations of the American Society of Hematology. The American Society of Hematology ITP Practice Guideline Panel. Ann Intern Med 1997;126:319-26.

2. Ursavas A, Ediger D, Ali R et al. Immune thrombocytopenia associated with pulmonary tuberculosis. J Infect Chemother 2010. [Epub ahead of print].

3. Jurak SS, Aster R, Sawaf H. Immune thrombocytopenia associated with tuberculosis. Clin Pediatr 1983;22:318-9.

4. Tsuro K, Kojima H, Mitoro A et al. Immune thrombocytopenic purpura associated with pulmonary tuberculosis. Intern Med 2006;45:739-42. 